\title{
10 Die Urheberrechtsreformen von 2003 und 2008
}

Die Reform von 2003 wird als Erster Korb und die von 2008 als Zweiter Korb bezeichnet. Die dort im Gesetz verankerten Regelungen für den Umgang mit Wissen und Information bezogen sich überwiegend auf Bildung und Wissenschaft. Dies trifft vor allem für den 2003 eingeführten § 52a zu, der verschiedentlich, aber doch irreführend schon als (allgemeine) Wissenschaftsschranke bezeichnet wurde. Auch andere Regelungen, wie die in den $\S \S 52 \mathrm{~b}$ und 53a, die den Spielraum von Vermittlungsorganisationen wie Bibliotheken an die Möglichkeiten der Informations- und Kommunikationstechnologien und die Erwartungen ihrer Nutzer anpassen sollten, sind in erster Linie auf Bildung und Wissenschaft bezogen. Diese Paragraphen sind mit den 2018 Gesetz gewordenen neuen $\S \S 60 \mathrm{a}-60 \mathrm{~h}$ des UrhWissG aufgehoben worden (vgl. Kap. 13). Das heißt nicht, dass alle Regelungen dieser Normen nun gänzlich ungültig geworden sind. Viele davon bestehen in den neuen Paragraphen zum Teil weiter bzw. wurden modifiziert und damit auch etwas praxistauglicher bzw. rechtssicherer gemacht. Diese alten Paragraphen (52a, 52b und 53a) sind im Anhang dokumentiert.

Daher wird hier auch auf diese Reformen des Ersten und Zweiten Korbs in der gebotenen Kürze eingegangen (ausführlicher vgl. Kuhlen 2008) ${ }^{306}$ Auch gestrichene Paragraphen des Gesetzes sind keineswegs nur der Schnee von gestern. Sie gehören zur Geschichte des Wissenschaftsurheberrechts in Deutschland. Aber es ist nicht nur die Geschichte, die hier interessiert. Vielmehr können gerade aus der Gesetzgebung des Ersten und Zweiten Korbs bzw. aus den der Gesetzgebung vorausgegangenen Auseinandersetzungen und aus den dann folgenden gerichtlichen Entscheidungen zu strittigen Auslegungsfragen die meisten der in Kap. 9 zusammengestellten Thesen bestätigt werden. Die Thesen (Kap. 9) behalten im Übrigen ihre Gültigkeit bis hin zur letzten Reform des UrhWissG und werden daher die Diskussion bis zum Ende dieses Textes begleiten. Die Defizite der im Ersten und Zweiten Korb verabschiedeten, auf Bildung und Wissenschaft bezogenen Normen werden im Folgenden zusammengefasst. ${ }^{307} \mathrm{Im}$ Mittelpunkt stehen dabei in erster Linie $\S 52 \mathrm{a}$ aus dem Ersten Korb und die $\S \S 52 \mathrm{~b}$ und 53a aus dem Zweiten Korb:

Vor allem in den $\S \S 52 \mathrm{a}$ und 52b haben unbestimmte Rechtsbegriffe wie „veröffentlichte kleine Teile eines Werkes”, „Werke geringen Umfangs“ sowie „einzelne

306 (Kuhlen 2008) Erfolgreiches Scheitern.

307 Die Defizite der Reformen 2003 und 2008 aus Sicht der Länder (Pflüger Zum 2016, 487): „ein Flickenteppich nicht aufeinander abgestimmter Schrankenregelungen“, „Vielzahl unklarer Rechtsbegriffe“; zudem: „rechtstatsächlich eine weitgehende Marginalisierung des Schrankenregimes der Körbe 1 und 2“.

Ә Open Access. (C) 2020 Rainer Kuhlen (c))BY This work is licensed under a Creative Commons Attribution 4.0 License. https://doi.org/10.1515/9783110693447-010 
Beiträge aus Zeitungen oder Zeitschriften“ große Rechtsunsicherheit bewirkt. Auch haben viele Einzelregelungen in ihnen zu Klagen der Verleger gegen nach ihrer Meinung zu weitgehenden Nutzungsangeboten der Bibliotheken geführt: Diese Verfahren erstreckten sich i. d. R. über viele Jahre, bis sie dann vom BGH und zum Teil sogar erst durch den EuGH entschieden bzw. dann an die zuständigen Landesoder Oberlandesgerichte zurückverwiesen wurden. Als unglücklich erwiesen sich auch, wie schon in 2.3 erwähnt, vage Formulierungen wie „Veranschaulichung im Unterricht“, wobei die Präposition „im“ von Kritikern des §52a oft wortwörtlich genommen wurde, bis dann (nach mehr als 10 Jahren) durch den BGH geklärt wurde, dass im Grunde „des“ Unterrichts gemeint sei, weil sonst die gesamte Regelung keinen Sinn ergebe. Seitdem ist klar, dass auch Vor- und Nachbereitung des Unterrichts sowie Prüfungsvorbereitungen durch §52a mit gedeckt sind, und so wurde das schließlich auch im UrhWissG 2018 verstanden.

Ob Folgehandlungen wie Speichern und Ausdrucken von digitalen Materialien, die von den Bibliotheken entsprechend § 52b bereitgestellt wurden, erlaubt waren oder nicht, war lange Zeit ebenfalls ungewiss. Klagen, die Verlage gegenüber entsprechenden Nutzungshandlungen von Bibliotheken (letztlich erfolglos) angestrengt hatten, gingen ebenfalls durch alle Instanzen über mehrere Jahre, bis dann im Zusammenspiel von BGH und EuGH entschieden wurde, dass die Berücksichtigung solcher Folgehandlungen im Urheberrecht vorgesehen werden könnten (vgl. 10.1.3).

Viele Regelungen trugen schon zur Entstehungszeit dem technologischen Stand nicht Rechnung, wie z. B., dass für den Kopienversand für Bibliotheken, wie schon zu Beginn erwähnt, nur grafische Dateien (Faksimile) verschickt werden dürfen (entsprechend §53a), also keine voll-digitalen Dateien, welche die Weiterverarbeitung oder Volltextsuche erlauben würden. Es hat dann noch weitere 15 Jahre gedauert, bis dies im UrhWissG 2018 aufgegeben wurde. Zu den Regelungen, die entgegen den technologischen Potenzialen formuliert sind, die aber nach Ansicht des Gesetzgebers durch die Vorgaben der EU als zwingend angesehen wurden, gehörte auch die Vorschrift in $\S 52 \mathrm{~b}$, dass digitalisierte Objekte aus dem Bestand der jeweiligen Bibliothek nur an den Leseplätzen (!) in den Räumen der Bibliothek eingesehen werden dürfen - obgleich heute jeder berechtigte Nutzer über die Netzwerke von Orten seiner Wahl (z. B. über VPN) Zugriff haben könnte bzw. sollte. Diese Einschränkung hat weiter bis heute Bestand, obgleich schon in der Planung der neuen EU-Urheberrechtsrichtlinie von 2016 (beschlossen dann 2019) deutlich wurde, dass die EU-Kommission Zweifel an der Berechtigung dieser unzeitgemäßen Restriktion hatte. Unklar blieb, ob und inwieweit vertragliche Lizenzangebote Priorität gegenüber den Nutzungserlaubnissen in den Schrankenregelungen haben sollen. Unklar ist auch geblieben, in welcher Form (pauschal oder individuell) die in den Normen vorgesehenen Vergütungen geregelt werden 
sollen. ${ }^{308}$ Beides wurde erst 2018 mehr oder weniger verbindlich entschieden. Die meisten dieser Einschränkungen der Schrankenregelungen, die aus heutiger Sicht in ihrer Kleinlichkeit und Unzeitgemäßheit schwer nachvollziehbar sind, hätten mit etwas mehr kreativer Hermeneutik vermieden werden können. Tatsächlich sind sie aber wohl in erster Linie der Sorge der Verleger bzw. dem intensiven und weitgehend erfolgreichen Lobbying ihrer Interessenvertreter quer durch alle Medien geschuldet - vor allem dem Lobbying des traditionell die Printmedien repräsentierenden Börsenvereins des deutschen Buchhandels. Sie wollten sich gegen die ihrer Ansicht nach zu intensive, genehmigungsfreie Nutzung der von ihnen auf den Markt gebrachten Werke wehren.

\section{1 §52a UrhG - ein unzureichender Versuch für eine Wissenschaftsschranke}

Im Zentrum des Ersten Korbs (Gesetz geworden am 13.9.2003 ${ }^{309}$ ) stand aus Sicht von Bildung und Wissenschaft $§ 52$ a Öffentliche Zugänglichmachung für Unterricht und Forschung (Text in 17.2.1). § 52a wurde trotz zum Teil heftiger Kritik an einigen Detailregelungen in der weiteren Fachöffentlichkeit durchaus als EU-weite erste Umsetzung von InfoSoc 2001 Art, 5, 3, a gewürdigt. ${ }^{310} \S 52$ a wurde als Versuch verstanden, über eine Schrankenregelung zugunsten von Bildung und Wissenschaft den veränderten Rahmenbedingungen der Digitalisierung für so gut wie allen Prozesse von Wissen und Information Rechnung zu tragen.

\subsection{1 §52a hätte auch ganz anders aussehen können}

Wäre die parlamentarische Beratung tatsächlich der ersten Vorlage für §52a durch den RefE des damaligen BMJ gefolgt, dann hätte §52a wirklich die Chance gehabt,

308 Die Kontroverse um pauschale Vergütung oder einzelfallbezogene Nutzung hatte Ende $2016 \mathrm{zu}$ einem heftigen Konflikt an den Hochschulen geführt, der vorübergehend durch die Rücknahme des zwischen KMK und VG WORT abgeschlossenen Rahmenvertrags zunächst einmal ausgesetzt wurde. Dieser Rahmenvertrag hatte eine Individualvergütung vorgesehen, die von den Hochschulen als unzumutbar zurückgewiesen wurde. Dieser Streit ist jetzt durch das UrhWissG zugunsten der Pauschalvergütung (zumindest vorübergehend) entschieden worden (vgl. 8.6).

309 Gesetz im Bundesanzeiger 10.9.2003 - https://bit.ly/2sieBiq.

310 "3. Member States may provide for exceptions or limitations to the rights provided for in Articles 2 and 3 in the following cases: (a) use for the sole purpose of illustration for teaching or scientific research, as long as the source, including the author's name, is indicated, unless this turns out to be impossible and to the extent justified by the non-commercial purpose to be achieved" - InfoSoc 2001 - https://bit.ly/3a600pL. 
als erste umfassende Wissenschaftsschranke, als „Prototyp einer Bildungs- und Wissenschaftsschranke" ( ZUM 2016, 485) zu gelten. Hier der sehr knappe, aber umfassende Vorschlag des damaligen BMJ:

§52a Öffentliche Zugänglichmachung für Unterricht und Forschung

(1) Zulässig ist, veröffentlichte Werke

1. zur Veranschaulichung im Unterricht ausschließlich für den bestimmt abgegrenzten Kreis von Unterrichtsteilnehmern oder

2. ausschließlich für einen bestimmt abgegrenzten Kreis von Personen für deren eigene wissenschaftliche Forschung öffentlich zugänglich zu machen, soweit die Zugänglichmachung zu dem jeweiligen Zweck geboten und zur Verfolgung nicht kommerzieller Zwecke gerechtfertigt ist.

Die gesamte Bundesregierung (Koalition SPD, Grüne/Bündnis 90) schloss sich dem in ihrer ersten Vorlage am 16. August 2002 an. Es kam dann aber nach den parlamentarischen Beratungen, vor allem denen im Rechtsausschuss, ganz anders. Aus den „Werken“ wurde dann „veröffentlichte kleine Teile eines Werkes, Werke geringen Umfang sowie einzelne Beiträge aus Zeitungen oder Zeitschriften“. Solche Einschränkungen, wie auch die enge Interpretation von „im Unterricht“ (also nicht den Unterricht begleitend) und das Verbot von Folgehandlungen wie Drucken und Speichern entsprachen dem damaligen, auf Verknappung und auf Schutz der kommerziellen Verwertung ausgerichteten Zeitgeist.

Man kann keineswegs davon ausgehen, dass die Juristen im damaligen BMJ sich der unionsrechtlichen Vorgaben aus der InfoSoc Richtlinie von 2001 nicht bewusst gewesen wären. Einige von ihnen waren ja über lange Jahre an der Entstehung dieser EU-Richtlinie selbst aktiv beteiligt. Sie haben in voller Kenntnis dieser Richtlinie ihren Vorschlag in die parlamentarische Debatte eingebracht. Das seitdem immer wieder vorgebrachte Argumente aus der Politik, „man würde ja gerne größere Nutzungsfreiheiten für Bildung und Wissenschaft erlauben, allein die EU-Vorgaben erlaubten das nicht" kann angesichts dieser Vorgabe des RefE nicht überzeugen. Für die nationale positive Gesetzgebung hat es an vielen Stellen bezüglich der Umsetzung von EU-Vorgaben großer Spielraum gegeben ("left ample manoeuvre for the Member States” - FN 311). Die EU-Kommission wies selbst, allerdings erst 2016, ${ }^{311}$ mit Hinweis auf eine von ihr in Auftrag gegebene Studie darauf hin, dass Einschränkungen (wie im deutschen UrhG dann bei §52a erfolgt) durch die Schranke InfoSoc 2001 Art. 5, 3, a nicht zwingend sind:

311 In dem Dokument Commission Staff Working Document Impact Assessment on the modernisation of EU copyright rules (Brussels, 14.9.2016 SWD(2016) 301 final). Accompanying the document Proposal for a Directive of the European Parliament and of the Council on copyright in the Digital Single Market. Part 2/3. 
The open-ended content of [this exception] left ample manoeuvre for the Member States to enact the conditions under which the exception could be enjoyed. Nothing prevents domestic laws to further define the beneficiaries, the types and quantities of works that can be used, as well as the type of use..$^{312}$

Aus den EU-Vorgaben, wie in diesem Fall InfoSoc 2001 Art. 5, 3 a, sind also die oben erwähnten Einschränkungen in §52a nicht zwingend abzuleiten. Auch zeigten die der Verabschiedung folgenden gerichtlichen Auseinandersetzungen, dass keineswegs die in $\S 52 \mathrm{a}$ (ebenso in 52b und 53a) festgeschriebenen Annahmen wie in Stein gemeißelt gültig sein müssen. Spektakulär und folgenreich war vor allem das Urteil des BGH vom 28.11.2013 (I ZR 75/12 - Meilensteine der Psychologie), durch das der langjährige Rechtsstreit (Landgericht Stuttgart vom 27. September 2011 und Oberlandesgerichts Stuttgart vom 4. April 2012) zwischen dem klagenden Meiner-Verlag und der Fernuniversität Hagen entschieden wurde.

\title{
10.1.2 Das Ende des „im“ in §52a-Alt
}

Gesetze dürfen nicht immer wortwörtlich für bare Münze genommen werden. $\mathrm{Ob}$ die Formulierung „Veranschaulichung im Unterricht“ nur durch ein Versehen oder auf Grund von erfolgreichen Lobbying aus Verlagsinteresse ins Gesetz genommen wurde, ist nicht mehr festzustellen. Auf jeden Fall wurde über 10 Jahre hinweg um die Präpositionspriorität gestritten. Dem allen hat der BGH schließlich ein Ende bereitet und damit auch den gesetzlichen Weg für das fast 15 Jahre spätere UrhWissG 2017/2018 geöffnet:

\begin{abstract}
Das „im Unterricht“ ist allerdings [... ] nicht durch die zeitlichen und räumlichen Grenzen des Unterrichts beschränkt, sondern kann sich auf andere Zeiten (wie die Vor- oder Nachbereitung des Unterrichts) und Orte (etwa den häuslichen Arbeitsplatz) erstrecken. [...] Im Übrigen wäre die Vorschrift praktisch bedeutungslos, wenn ein Zugänglichmachen nur während des Unterrichts zulässig wäre. ${ }^{313}$
\end{abstract}

Eine bloß semantische Interpretation einer Präposition reicht nicht aus, um den Sinn eines Gesetzes zu verstehen. Das leistet besser eine teleologischen Auslegung das ist entsprechend der Semiotik die pragmatische Dimension von sprachlichen Ausprägungen auch in Gesetzen. Allzu groß oder weiterführend war diese Umin-

312 (Triaille et al. 2103) Study on the application of Directive 2001/29/EC on copyright, S. 380. 313 So sensationell ist das nicht, denn nach der Definition der öffentlichen Zugänglichmachung in $\S 19$ a UrhG, welche ja gerade auch in §52a erlaubt sein soll, muss ,der Abruf urheberrechtlich geschützter Werke durch die Nutzer jederzeit und an jedem Ort möglich sein“ (Hoeren 2011) Kleine Werke?, hier: S. 371. 
terpretation des „im“ zum „des“ oder „für“ aber dann doch nicht. Hätte bei der Gelegenheit nicht auch der Schritt von der didaktisch unzeitgemäßen, exklusiv lehrebezogenen Einschränkung „zur Veranschaulichung des Unterrichts“ zu einer zeitgemäßen Öffnung getan werden könne, nämlich für nicht kommerzielle Zwecke von Lehr- und Lernprozessen an Bildungseinrichtungen (vgl. Kreutzer/Hirche 2017)?314 Nicht nur das Lehren, sondern auch das Lernen sollte durch die Urheberrechtsschranke unterstützt werden. Lernen wird weiter von den anregenden Vorgaben der Lehrenden unterstützt. Aber zunehmend ermuntern oder fordern sogar Lehrende, besonders bei E-Learning- und Fernkursen, das selbständige Recherchieren und Einbringen einschlägiger Literatur. Im Sinne des kollaborativen Lernens (Kerres/Jechle 2001) ${ }^{315}$ ist es bei solchen Kursen auch erwünscht, dass nicht nur der recherchierende Lernende davon Nutzen zieht, sondern dass er die Ergebnisse seiner Recherchen auch in den elektronischen Pools des Kurses einspeist und damit für allen anderen in dem Kurs zugänglich macht.

\subsubsection{Der Streit um die akzessorischen Rechte des Druckens und Speicherns}

Vergleichbar der Diskussion um „,im Unterricht“ oder „für den Unterricht“ bzw. „,des Unterrichts" wurde auch intensiv darüber gestritten, ob die erlaubt einsehbaren Werke oder Teile von ihnen ausgedruckt und/oder gespeichert werden dürfen. Speichern oder Drucken, sogenannte akzessorische Rechte, sind selbstverständliche zeitgemäße Grundfunktionen beim Umgang mit Wissen und Information. Wenn eine Schranke, wie §52a, die öffentliche Zugänglichmachung (im definierten Umfang) zulässt, dann sollten, so sollte man annehmen, auch die entsprechenden Folgehandlungen erlaubt sein, auch wenn diese im Gesetzestext nicht explizit erwähnt sind. Aber hier ist die Hürde einer an sich naheliegenden teleologischen bzw. pragmatischen Interpretation des Gesetzes etwas höher als bei dem „Im“-Problem. Auch hier hat es mehr als 10 Jahre gedauert, bis dann über den BGH, nach unterschiedlichen Rechtsauslegungen durch die beteiligten Landes- und Oberlandesgerichts, eine verbindliche Klärung zugunsten der Erlaubnis der erwähnten Folgehandlungen zustande kam. Allerdings fiel die Klärung bei §52a etwas anders aus als für $\S 52 \mathrm{~b}$ (s. unten).

Mit Blick auf §52a hatte ein Verlag (Kröner) gegen eine als zu weitgehend angesehene Auslegung von §52a durch die Fernuniversität Hagen geklagt. Kröner war der Ansicht, dass die Bibliotheken für ihre digitalen Semesterapparate dafür

314 (Kreutzer/Hirche 2017) Rechtsfragen zur Digitalisierung in der Lehre.

315 (Kerres/Jechle 2001) Didaktische Konzeption des Tele-Lernens. Das UrhWissG hat 2018 in $\S 60 a$, Abs. 1, 1 schließlich auch die Teilnehmer an Lehrveranstaltungen begünstigt. (vgl. 13.2.1) 
Sorge hätten tragen müssen, dass die nach $\S 52 \mathrm{a}$ bereitgestellten Materialien durch die Studierenden weder ausgedruckt noch gespeichert werden können. In seinem Urteil von November $2013^{316}$ befand der BGH, dass auch bei Folgehandlungen entsprechend §52a das Vervielfältigen (durch Ausdrucken und Speichern) erlaubt ist. Zunächst räumte der BGH ein, dass §52 nur das Zugänglichmachen explizit erlaubt sei, aber nicht das Vervielfältigen. Aber das bedeute nicht, dass ein Zugänglichmachen nicht zulässig ist, wenn eine anschließende Vervielfältigung ermöglicht wird. §52a sagt nichts über Vervielfältigungen aus (weder dafür noch dagegen). Gegen Vervielfältigen wurde vor allem der Drei-Stufen-Test ins Spiel gebracht. Drucken und Speichern verstoße, so in der Klage, gegen die zweite Stufe des Tests, also gegen die normale kommerzielle Verwertung. Der BGH sah allerdings das nicht als gegeben an, da die normale Verwertung durch die Beschränkung der Zugänglichmachung auf kleine Teile in §52a nicht beeinträchtigt werde. ${ }^{317}$ Der BGH war sich in seiner Entscheidung so sicher, dass er keine Notwendigkeit sah, den EuGH um Klärung der unionsrechtlichen Kompatibilität zu bitten. Zu einer endgültigen Entscheidung im gesamten Fall - der BGH hatte das Verfahren an das Revisionsgericht in Stuttgart zurückverwiesen - ist es übrigens nicht mehr gekommen. Das UrhWissG hatte dann die Meinung des BGH bei §60a und §60c und teilweise auch bei §60e mit etwas erweiterten Einschränkungen übernommen (vgl. Abschnitte 13.2.1, 13.2.3 und 13.2.5); trotzdem, ein kleiner Zwischensieg für kreative Hermeneutik. ${ }^{318}$

Bei §52b-Alt kam der BGH bezüglich des Druckens und Speicherns zu einer etwas anderen Einschätzung, auf die hier, vor allem wegen der Begründung, die fern von jeder wissenschaftlichen Praxis war, noch etwas näher eingegangen werden soll. Anlass des ersten BGH-Urteil von 2013 in dieser Sache (nach vorangegangenen Urteilen des Landgerichts Frankfurt und die darauffolgende Revision vom 16.3.2011) war eine Klage (Ulmer vs. TU Darmstadt - Elektronische Leseplätze) gegen die Reichweite der durch die Universitätsbibliothek ermöglichten Nutzung zunächst von §52b-Alt UrhG. Der BGH hatte dann aber 2013 sein Verfahren erst einmal ausgesetzt und zur Lösung der dabei aufgetretenen unionsrechtlichen

\footnotetext{
316 BGH I ZR 76/12 vom 26.11.2013 - https://bit.ly/2Mt37Bg.
}

317 Der BGH verstand kleine Teile als $12 \%$ des Gesamttextes, allerdings höchstens nur 100 Seiten. 318 Allerdings wurde in dem Urteil des BGH auch zunächst festgeschrieben, dass die öffentliche Zugänglichmachung (z. B. auf Lernplattformen) gemäß §52a Abs. 1 Nr. 1 UrhG nicht geboten sei, wenn der Hochschule vom Rechtsinhaber eine angemessene Lizenz für die fragliche Nutzung angeboten wurde. Das allerdings wurde 2018 vom Gesetzgeber nicht so gesehen. Er räumte über $\S 60$ e Schrankenregelungen den Vorrang vor vertraglichen Angeboten ein. Aber auch das wird in den Nachfolgediskussionen über Planungen zu einer Lizenzierungsplattform wieder auf die Tagesordnung kommen (zur Lizenzierungsplattform vgl. 13.7.2). 
Probleme der EuGH um Klärung gebeten. Das geschah dann 2014. Der BGH folgt der EuGH-Einschätzung 2015. Der Klage wurde in den wesentlichen Teilen nicht stattgegeben.

Aus den vielfältig dabei behandelten Problemen ${ }^{319}$ wird im Folgenden vor allem auf die Annexhandlungen des Druckens und Speicherns eingegangen. Dass der BGH eine Normübertragung durchaus elegant-kreativ zu nutzen verstanden hat, zeigte sich an diesem Streit um diese akzessorischen Rechte. Auch in §52b war nur von Zugänglichmachung die Rede ist, also vom Recht der Bibliothek auf (elektronische) Wiedergabe von Werken, die sie aus ihrem Bestand digitalisiert hatte. Strittig war nun in der Klage, ob durch $\S 52 b$ die akzessorische Rechte des Speicherns und Ausdruckens als erlaubt anzusehen sind. Die Bibliothek der TU Darmstadt sah sich berechtigt, den Nutzern die Möglichkeit zu geben, das Werk ganz oder teilweise auf Papier auszudrucken bzw. auf einem USB-Stick zu speichern, so dass sie nicht nur auf die Ansicht des Textes vor Ort angewiesen waren, sondern ihn in analoger oder elektronischer Form mit sich nehmen konnten, wohin auch immer sie wollten.

In dem ersten BGH-Urteil zu §52b vom 20.9.2012 hatte der BGH Bedenken gegenüber dem Speichern von vollständigen digitalisierten Werken geäußert und dieses untersagt. Aber das Ausdrucken schien ihm erlaubt zu sein. Wie der BGH zu dieser Differenzierung von Drucken und Speichern (ersteres erlaubt, zweites untersagt) gekommen ist, ist ein Musterbeispiel dafür, dass Gerichten die Nutzungsgewohnheiten und die Praxis des wissenschaftlichen Arbeitens eher fremd sind..$^{320}$ Das soll hier vollständig zitiert werden:

319 Geklärt werden musste z. B. auch das Problem, ob Bibliotheken überhaupt das Recht haben, Werke aus ihrem Bestand zu digitalisieren. In der EU-Vorgabe in Art. 5 Abs. 3 lit. n InfoSoc 2001 war nur von „öffentlicher Wiedergabe“ die Rede. Das erschien dem BGH 2013 so schwierig, dass er den EuGH die Frage stellt, ob diese Digitalisierung mit der unionsrechtlichen Vorgabe) kompatibel sei. Der EuGH bejahte diese Frage mit teleologischer Begründung: „Dieses Recht zur Wiedergabe von Werken, das den in Art. 5 Abs. 3 lit.n der Richtlinie 2001/29 genannten Einrichtungen wie öffentlich zugänglichen Bibliotheken in den tatbestandlichen Grenzen dieser Bestimmung zusteht, drohte einen großen Teil seines sachlichen Gehalts und sogar seiner praktischen Wirksamkeit zu verlieren, wenn diese Einrichtungen kein akzessorisches Recht zur Digitalisierung der betroffenen Werke besäßen. “ Der BGH übernahm das dann fast wortwörtlich in seiner Endentscheidung von 2015 (Urteil des I. Zivilsenats vom 16.4.2015 - I ZR 69/11).

320 Thomas Hartmann bezeichnete es in seinem Kommentar zu diesem 52b-BGH-Urteil BGH als „ungewöhnlich, wenn der $B G H$ bestimmte wissenschaftliche Nutzungen als unerlässlich, andere als nachgeordnet bewertet. “ In diesem Fall Ausdrucken als unerlässlich erlaubt - Speichern als nachgeordnet und nicht erlaubt. Vgl. EuGH-Vorlage zur Zulässigkeit elektronischer Leseplätze in Bibliotheken - Elektronische Leseplätze mit einer Anmerkung von Thomas Hartmann. GRUR 2013, S. 513 . 
Die Bestimmung des Art. 5 III lit. n der Richtlinie 2001/29/EG soll es den in dieser Regelung genannten Einrichtungen gestatten, die Werke ihres Bestands zu Zwecken der Forschung und privater Studien zugänglich zu machen. Ein wissenschaftliches Arbeiten mit Texten ist nach heutigem Verständnis in der Regel nur dann in zweckentsprechender Weise möglich, wenn wichtige Passagen eines Textes markiert und mit Anmerkungen versehen und die entsprechenden Textauszüge zum weitergehenden Studium aus der Bibliothek mitgenommen werden können. Ein sinnvolles Arbeiten mit auf Terminals wiedergegebenen oder zugänglich gemachten Texten zu Zwecken der Forschung und privater Studien setzt daher die Möglichkeit zum Ausdrucken und Bearbeiten der Texte voraus. ${ }^{321}$

Vor allem die Begründung des BGH für das Ausdrucken geht an der in Bildung und Wissenschaft tatsächlichen und selbstverständlich gewordenen Praxis der Arbeit mit elektronischen Texten vorbei. Sollte sich nicht auch ein BGH auf die sich in elektronischen Räumen längst entwickelte Wissenschafts- und Lehr- und Lernpraxis einlassen? Am Ende war sich der BGH offensichtlich nicht sicher, ob Drucken in der Auslegung des deutschen $\S 52 \mathrm{~b}$ wirklich mit dem Unionsrecht verträglich ist und ob vielleicht Speichern doch erlaubt sein sollte, und stellte, neben anderen Fragen, die folgende Frage an den EuGH: „Dürfen die von den Mitgliedstaaten gem. Art. EWG_RL_2001_29 Artikel 5 EWG_RL_2001_29 Artikel 5 Absatz III lit. n der Richtlinie 2001/29/EG vorgesehenen Rechte so weit reichen, dass Nutzer der Terminals dort zugänglich gemachte Werke auf Papier ausdrucken oder auf einem USB-Stick abspeichern können?“

Der EuGH ${ }^{322}$ beantwortete die Frage diplomatisch und kreativ zugleich. Nach seinem Verständnis von Art. 5 Abs. 3 lit. n der weiterhin gültigen InfoSoc-Richtlinie von 2001 könne aus dem Recht der Wiedergabe (wie in §52b vorgesehen) zwar nicht das akzessorische Recht der Vervielfältigung hergeleitet werden kann. Drucken und Speichern sind im Urheberrecht Vervielfältigungsvorgänge und ,sind nicht erforderlich, um dieses Werk den Nutzern auf eigens hierfür eingerichteten Terminals unter Einhaltung der in diesen Bestimmungen festgelegten Voraussetzungen zugänglich zu machen“ (RN 54). Allerdings, so folgert der EuGH aus Art. 5 Abs. 2 lit. a oder b der InfoSoc-Richtlinie, könnten solche Handlungen wie Drucken und Abspeichern „durch die nationalen Rechtsvorschriften zur Umsetzung der Ausnahmen und Beschränkungen dieser Richtlinie gestattet sein, sofern im Einzelfall die in diesen Bestimmungen festgelegten Voraussetzungen erfüllt sind“ (RN 57). Das ist durchaus generalisisierbar - eine wichtige Aufforderung auch an den deutschen Gesetzgeber, von seinem großen Spielraum bei der Formulierung positiver Gesetze weitgehend und der Praxis angemessen Gebrauch zu machen.

321 BGH GRUR 2013, 506, Tz. 30.

322 EuGH Rechtssache C-117/13 vom 11.9.2014 - https://bit.ly/1G7KBRG. 
Der BGH wollte offenbar darauf nicht warten. Er hat im April $2015^{323}$ die Ausführungen des EuGH weit ausgelegt und ist in dieser Sache selbst aktiv geworden und hat damit sozusagen die politische Initiative übernommen. Direkt auf Formulierungen aus $\S 52 \mathrm{~b}$ konnte er sich wohl nicht berufen. Aber er sah keine Notwendigkeit, dass die Bibliotheken von sich aus die Möglichkeit von Ausdrucken und Speichern aktiv verhindern, also die Terminals so restriktiv ausstatten müssten, dass Drucken und vor allem Speichern nicht möglich ist. Diese Lösung des Problems wurde durch die Zusammenschau von zwei Normen möglich. Vervielfältigungen, also auch Drucken und Speichern elektronischer Materialien, sind nach §53 UrhG zum privaten Gebrauch erlaubt. ${ }^{324}$ Vorrang hatte für den BGH offensichtlich $\S 53$ Privatkopie, der solche Vervielfältigungshandlungen erlaubt. ${ }^{325}$

Gegen das Urteil des BGH wurde schweres Geschütz aufgefahren. Der Börsenverein kritisierte den Tag der Entscheidung als „schwarzen Tag für Forschung und Lehre an deutschen Hochschulen ... Für die Verlage komme diese Auslegung des Urheberrechtsgesetzes einer vollständigen Enteignung gleich. “326 Es werde geprüft, ob zusammen mit dem Verleger Ulmer eine Verfassungsbeschwerde eingelegt werden sollte. Entsprechend einem Artikel in buchreport vom 21.10.2015 erhob Ulmer tatsächlich eine Verfassungsbeschwerde gegen das BGH Urteil: „Dass Autoren heute dulden müssen, dass Richter in ihre Grundrechte eingreifen und weder die selbstverständliche Abwägung über die Notwendigkeit und Gebotenheit einer Enteignung vornehmen noch den Enteigneten dafür eine Entschädigung zusprechen, ist nicht hinnehmbar." ${ }^{327}$ Wie die meisten Verfassungsbeschwerden wurde auch diese aber nicht weiterverfolgt.

Einiges von dem BGH-Urteil ist inzwischen nur Geschichte: $\S 53$ Abs. 2, 1, das Recht der Privatkopie zum „eigenen wissenschaftlichen Gebrauch“, ist durch das zum 1.3.2018 gültig gewordene UrhWissG gestrichen worden, zudem die Erlaubnis zur Vervielfältigung von Zeitungsbeiträgen in $\S 53$, (2), 4, a. Neue Nutzungsregelungen für Wissenschaft und Bildung sind nun in den beiden $\$ \S 60 \mathrm{a}$ und 60c festgelegt, welche Teile aus $\S \S 53$, 52a und 52b modifizierend, erweiternd, aber auch

323 Urteil des I. Zivilsenats des BGH vom 16.4.2015 - I ZR 69/11 - https://bit.ly/2Zgo9Jh.

324 „Ein Ausdrucken oder Abspeichern von an elektronischen Leseplätzen bereitgestellten Werken kann in vielen Fällen als Vervielfältigung zum privaten oder sonstigen eigenen Gebrauch nach $\S 53$ UrhG zulässig sein.“ (ebda. FN 323).

325 Allerdings sehr viel gewonnen war dadurch auch nicht: Die Vervielfältigungshandlungen zum eigenen Gebrauch sind entsprechend §53, (2), 4, a und b, nur erlaubt für „,kleine Teile eines erschienenen Werkes“ (das aber nur, wenn es schon mehr als zwei Jahre vergriffen ist), aber dann doch für ganze Beiträge in Zeitschriften oder Zeitungen.

326 Kritik des Börsenvereins an BGH-Urteil zum §52b. Börsenblatt 17.4.2015 - https://bit.ly/31NoiBJ. 327 Ulmer erhebt Verfassungsbeschwerde. Quelle: buchreport 21.10.2015 - https:// /2MsceSP. 
eingrenzend integrieren. Speichern und Ausdrucken sind durch das UrhWissG in den auf Bildung und Wissenschaft bezogenen Schrankenregelungen erlaubt allerdings ist das immer wieder mit Einschränkungen verbunden. Trotzdem auch dies ein kleiner verzögerter Sieg von kreativer Hermeneutik.

Zwischenfazit. Wie immer auch versucht wurde, sei es durch die Regelungen im Zweiten Korb oder 2017/18 durch die im UrhWissG, den Umfang der erlaubten Folgehandlungen wie Drucken oder Abspeichern festzulegen, das Ergebnis ist wenig tauglich für Praxis in Bildung und Wissenschaft und auch unbrauchbar für die Bibliotheken, die diese Festlegungen umsetzen müssen. Unbrauchbar mit der Vorgabe durch unbestimmte Rechtsbegriffe (2003 und 2008), aber auch unbrauchbar später im UrhWissG durch fixe Quantifizierungen (z. B. $15 \%$ eines Werks). Zudem, wie oder von wem soll kontrolliert werden, wenn ein Nutzer das ganze Buch abspeichert oder ausdruckt, obwohl nur kleine Teile oder genau $15 \%$ für den gegebenen Zweck zulässig sein sollen? Oder soll der Nutzer wirklich das Drucken abbrechen, wenn diese Prozentwerte erreicht sind? Oder muss der Bibliothekar einschreiten? Eine Beschränkung der Erlaubnis der Vervielfältigung auf einen Anteil des Werks ist gerade im Bereich Wissenschaft praxisfern, weil in deren Publikationen verbreitet mit Querverweisen gearbeitet wird, die es notwendig machen, auch in Fällen, in welchen das Interesse zunächst nur auf Teile eines Werks gerichtet ist, auf den vollständigen Text zurückzugreifen.

\subsubsection{Wie der erzwungene Gang in die Bibliothek hätte verhindert werden können}

Zum Abschluss der Diskussion um die Reformen 2003 und 2008 soll noch auf ein juristisch komplizierteres und besonders unzeitgemäßes Beispiel eingegangen werden, welches im Übrigen bis heute Bestand im UrhG hat. Durch $\S 52 \mathrm{~b}$ wurde den Bibliotheken das Recht der öffentlichen Zugänglichmachung für die Nutzung der Digitalisate aus ihren Beständen stark eingeschränkt. Die Norm spricht nur von „zugänglich machen“, nicht von „öffentlich zugänglich machen“. Der Unterschied, mehr als nur eine „dogmatische Feinheit” (Steinhauer ZUM 2016, 492), ist entscheidend, denn die öffentliche Zugänglichmachung ist (seit den WIPO-Verträgen) gerade dadurch definiert, dass die elektronische Nutzung von Orten und Zeiten der Wahl des Nutzers möglich ist. Das ist durch $\S 52 b$ keineswegs gegeben. Die Bibliotheksnutzer müssen in die Räumlichkeiten der Bibliotheken gehen, um die Digitalisate der Bibliothek einsehen zu können. Diese Beschränkung bleibt sogar bis in die Gegenwart in §60e des UrhWissG erhalten - obgleich dieser Ausschluss des externen Zugriffs angesichts der vollständigen telekommunikativen Vernet- 
zung aller Akteure in Bildung und Wissenschaft heute noch absurder ist und eine Verschwendung öffentlicher Mittel zur Folge hat. Für diese Beschränkung wird die Unions-Vorgabe der InfoSoc-Richtlinie von 2001 verantwortlich gemacht. ${ }^{328}$ 2001 war der deutsche Wissenschaftsrat schon vorausschauender. Er formulierte lapidar: „Bei digitalen Objekten entfällt die Zugriffsvoraussetzung der örtlichen Nähe. “329

Auch der BGH in seinem Urteil von April $2015^{330}$ blieb bei der Beschränkung der Nutzung auf „Terminals ${ }^{331}$ in den Räumen der Bibliothek“. So muss es wohl die Aufgabe der nicht-juristischen Öffentlichkeit der Zivilgesellschaft sein, der Politik und den Richtern verwertbare Hinweise darauf geben, dass der Zeitgeist und vor allem auch die technologischen Rahmenbedingungen sich geändert haben und neue pragmatische Interpretationen des Wortlauts von Gesetzen erlaubt sind. Schon zu Zeiten der Entstehung des $§ 52 b$ war es technisch für jedermann möglich, über VPN einen gesicherten Zugriff auf die elektronischen Materialien zu bekommen, welche die Bibliothek gekauft hat oder durch Lizenzvereinbarungen zur Nutzung „erworben“ hat. Das geschieht ja auch als alltägliche Praxis. Auch hierfür gibt es zuweilen noch Einschränkungen, wenn z. B. ein Verlag in den Lizenzvereinbarungen solche externen Zugriffe explizit ausschließt. ${ }^{332}$ Nicht nachzuvollziehen ist, dass ausgerechnet bei den Werken, die die Bibliotheken schon rechtmäßig in analoger Form zu bekommen, der externe Zugriff auf diese Werke in digitalisierter Form untersagt wird. Kreative juristische Hermeneutik ist gefragt, um dem Sinn einer Vorgabe gegenüber dem Wortlaut Geltung zu verschaffen. Sollte es nicht mehr als nur ein kleiner Sprung sein, das „in den Räumen der Bibliotheken“ auf die

328 InfoSoc 2001, RN 40: “Jedoch sollte diese Ausnahme oder Beschränkung [,,zugunsten bestimmter nicht kommerzieller Einrichtungen, wie der Öffentlichkeit zugängliche Bibliotheken und ähnliche Einrichtungen sowie Archive"] auf bestimmte durch das Vervielfältigungsrecht erfasste Sonderfälle begrenzt werden.“ Konkret durch Art. 5 Abs. 3 lit. n): „für die Nutzung von Werken und sonstigen Schutzgegenständen, für die keine Regelungen über Verkauf und Lizenzen gelten und die sich in den Sammlungen der Einrichtungen gemäß Absatz 2 Buchstabe c befinden, durch ihre Wiedergabe oder Zugänglichmachung für einzelne Mitglieder der Öffentlichkeit zu Zwecken der Forschung und privater Studien auf eigens hierfür eingerichteten Terminals in den Räumlichkeiten der genannten Einrichtungen“.

329 Wissenschaftsrat: Empfehlungen zur digitalen Informationsversorgung durch Hochschulbibliotheken“ 13.7.2001 (Drs. 4935/01), S.19 - https://bit.ly/2P5TbzJ.

330 Urteil des I. Zivilsenats des BGH vom 16.4.2015 - I ZR 69/11 - https://bit.ly/2Zgo9Jh.

331 Die Pressemitteilung des BHG spricht nach wie vor von „Leseplätzen“, obgleich inzwischen durch die Rechtsprechung klargestellt wurde, dass damit nicht nur das „Lesen“ gemeint sein kann. 332 Z. B. sind (derzeit) juristische Zeitschriften des Beck-Verlags nicht über VPN zu erreichen vermutlich weil Missbrauch vermutet wurde, wenn z. B. studentische Aushilfskräfte, die z. B. in Anwaltskanzleien arbeiten, über VPN ihr Zugriffsrecht in den Dienst der kommerziellen Nutzung durch die Kanzlei stellen würden. 
elektronischen Räume der digitalen Bibliotheken zu beziehen? Hätten die Gerichte und die Rechtswissenschaftler diesen Sprung gewagt, dann hätten Nutzer von ihrem Arbeitsplatz im elektronischen Raum aus die Dienstleistung der digitalen Bibliothek nutzen können und wären nicht mehr gezwungen gewesen, in die physisch vorhandenen Räume der Bibliothek zu gehen. ${ }^{333}$

Hier soll eine zweifache Hilfestellung für den Sprung gegeben werden, die erste eine (relativ schwache) semantische und die zweite eine starke technische. Zur Semantik: Im englischen Text von Art. 5 Abs. 3 lit. $n$ ist nicht von „Räumlichkeiten“ oder „Räumen“ die Rede, sondern von „on the premises“. Der Plural von „premise“ bedeutet in erster Linie „Grundstück“, auch „Räume“ und „Haus nebst Zubehör“. Viele der in analogen Welten entstandenen und anzuwendenden Begriffe, wie der physische Raum, haben unter den Bedingungen der Telemediatisierung ${ }^{334}$ eine virtuelle Bedeutung bekommen. Das Internet wird so auch als virtueller Raum oder als Gesamtheit der virtuellen telemediatisierten Räume verstanden.

Dieses Verständnis von Bibliotheken als virtuelle Räume sollte eine zeitgemäße Interpretation der Räumlichkeiten erlauben, dass Benutzer von ihrem Arbeitsplatz aus ihre elektronisch vernetzten Rechner so verwenden, als ob diese Endgeräte „on the premises“, auf dem Grundstück der Bibliothek wären. ${ }^{335}$ Das ist der Sinn eines VPN (virtual private network)-Anschlusses eines entfernten Rechners zur Quelle der Information. ${ }^{336}$ Das Übertragungsmedium löst sich, wie der Name VPN schon sagt, von dem physikalischen Übertragungsmedium und existiert nur noch virtuell. VPN ermöglicht gerade das, was durch $\S 52 \mathrm{~b}$ an sich vorgesehen sein sollte: Durch den durch VPN gegebenen Remote Access wird ein Nutzungsszenario

333 Eine zeitgemäße Gestaltung von Bibliotheksschranken sollte in Rechnung stellen, dass Wissenschaftler (sicherlich anders als Studierende) in den meisten Disziplinen so gut wie gar nicht mehr in die Bibliotheken gehen, geschweige denn real darin arbeiten. Das stellt keineswegs die Bedeutung der Bibliotheken in Frage (voll sind die Lesesäle überwiegend durch die Studierenden ohnehin). Gebraucht und genutzt werden weiter die vielfältigen Dienste und Leistungen der Bibliotheken, aber eben nicht vor Ort, in der Bibliothek, sondern remote, wo auch immer gerade gearbeitet wird.

334 Telemediatisierung ist ein Kunstwort, das Prozesse der Informatisierung, der Telematisierung/ Vernetzung und von Hypertextifizierung/Multimedia) zusammenfasst. Telemediatisiert sind die Prozesse der Systeme und Leistungen im Internet.

335 Dass dies auch praktisch geht, zeigt das Angebot der FU-Berlin, ihren Angehörigen über einen „ZEDAT-Account“ einen geschützten externen Zugang zu den E-Ressourcen der Bibliothek zu ermöglichen (s. FN 580).

336 Ein ähnlicher Dienst für den geschützten kontrollierten Zugang zu Informationen wird vom Deutschen Forschungsnetz mit DFN-AAI (https://www.aai.dfn.de/) - Authentifizierungs- und Autorisierungs-Infrastruktur angeboten, welche das international gebräuchliche Shibboleth-Programmpaket verwendet. 
aufgebaut, „bei dem Heimarbeitsplätze oder mobile Benutzer (Außendienst) in ein Unternehmensnetzwerk [auch in ein Wissenschaftsnetzwerk - RK] eingebunden werden. Der externe Mitarbeiter soll so arbeiten, als wenn er sich im Netzwerk des Unternehmens befindet.“337 Die durch VPN ermöglichte Verbindung ist ein geschützter Tunnel, bei dem die passwortgeschützte und verschlüsselte Kommunikation zwischen Nutzer und externer Quelle gegen Angriffe von außen geschützt ist. Solche Datenübertragungsdienste werden schon immer in und von den Gebäuden („on the premises“) einer Organisation bereitgestellt. Der Nutzer befindet sich sozusagen durch die VPN-Verbindung „on the premises“, und zwar besonders geschützt - geschützt von beiden Seiten, für den Nutzer und für die anbietende Bibliothek. Der Schutz vor dem Zugriff und Missbrauch unberechtigter Personen war wohl der Grund für die in der InfoSoc-Richtlinie vorhandene und in $§ 52 \mathrm{~b}$ übernommene Regelung des ,in den Räumen“. Sie war zweifellos nicht gedacht als Schutz des Interesses der Bibliotheken, dass möglichst viele Nutzer in die Räume der Bibliothek kommen.

Eine VPN-Übertragung mag tatsächlich schon ein größerer Sprung von den physischen Räumen der Bibliothek zu den virtuellen Räumen sein. Die Mehrheit der RechtswissenschaftlerInnen scheint die Beschränkung auf die physischen Räume zu akzeptieren, weil das durch die zitierte Bestimmung der InfoSoc-Richtlinie erzwungen oder zumindest nahegelegt wird. Allenfalls kommt ein Kommentar in der Literatur vor, diese Beschränkung sei „aus rechtspolitischer Sicht zu bedauern“. 338 All das mag aus der Sicht von 2001 oder auch noch von 2008 gerechtfertigt sein. Aber dass der Gesetzgeber heute noch im UrhWissG meint, den Nutzern der Dienste von Bibliotheken einen Zwang verordnen zu müssen, in die Räume der Bibliothek zu gehen, ist nicht nur skandalös, sondern hätte auch ein Fall für die Rechnungshöfe werden können. Schließlich hat die öffentliche Hand Millionen an Euro investiert hat, um die Hochschulen mit entsprechender Netzinfrastruktur zu versorgen, einschließlich der entsprechenden Struktur in und zu den Bibliotheken.

Der politische Wille für eine Öffnung der Nutzung, unabhängig von den physischen Räumen, war an sich seit gut 15 Jahren klar erkennbar. Wie schon in 3.5 zitiert, hatte die Politik im Strategischen Positionspapier des damaligen Bundesministeriums für Bildung und Forschung von 2002 zur Zukunft der wissenschaftlichen Information mit dem Titel „Information vernetzen - Wissen aktivieren“ die Leitidee des Zugriffs auf publiziertes Wissen ohne Beschränkung von Raum und Zeit aufgegriffen. Im ersten strategischen Ziel heißt es: „Den Zugang zur weltweiten wissenschaftlichen Information für jedermann zu jeder Zeit und von jedem Ort zu

337 VPN - Virtual Private Network. Quelle: Elektronik-Kompendium.de - https://bit.ly/2gdMmtr. 338 (Spindler 2008) Reform des Urheberrechts im „Zweiten Korb“, S. 13. 
fairen Bedingungen sicherstellen.“339 „Zu jeder Zeit und von jedem Ort“ - nicht nur zu den Öffnungszeiten der Bibliotheken und von den Leseplätzen in den Bibliotheken! Der politische Willen hätte sich auch gegenüber veralteten Vorgaben aus dem Unionsrecht mit etwas Kreativität durchzusetzen lassen.

\subsection{Unbekannte Nutzungsarten}

Im Zusammenhang des Zweiten Korbs wurden auch die Nutzungsrechte im UrhG Abschnitt 2 geändert, insbesondere die Regelung für die Nutzung bislang unbekannter Nutzungsarten. Dadurch wurde der bisherige Abs. 4 von $\S 31$ gestrichen und ein neuer $\S 31$ a eingefügt (im Zusammenhang mit $\S 1371$ Abs. 1). Abs. 4 lautete bis dahin: „Die Einräumung von Nutzungsrechten für noch nicht bekannte Nutzungsarten sowie Verpflichtungen hierzu sind unwirksam.“ Dies scheint auf den ersten Blick nur eine kleine Änderung zu sein. Bislang war es nicht möglich, dass der Urheber den Verwertern/Verlagen Nutzungsrechte für bis dahin unbekannte Nutzungsarten überträgt. In der Begründung der Bundesregierung für diese Öffnung heißt es: ${ }^{340}$ „Ferner soll angesichts der fortschreitenden technischen Entwicklung das bisher für den Urheber geltende Verbot, über noch unbekannte Nutzungsarten zu verfügen, gelockert werden. “ Tatsächlich stärkt die neue Regelung die Rechte der Urheber kaum - es sei denn, man interpretiert „Stärkung“ dadurch, dass der Urheber nun das Recht hat, die Nutzungsrechte an einen Verlag auch für solche Nutzungen zu übertragen, die zum Zeitpunkt der Übertragung noch unbekannt waren. Vorher war es durch $\S 31$ Abs. 4 überhaupt untersagt, dafür Nutzungsrechte einzuräumen. Das Verbot ist jetzt aufhoben, und jetzt hat jeder Autor ein erweitertes Übertragungsrecht. Gehört es also zur Freiheit eines Urhebers möglich viele seiner Rechte aufgeben zu dürfen? Tatsächlich handelt es sich mit der neuen Regelung nicht nur um eine Einschränkung der Rechte von Urhebern, sondern auch um eine Einschränkung des Gemeinfreiheitsinteresses der Öffentlichkeit. Das wurde von Rechtsetzung und Rechtsprechung allerdings anders gesehen. ${ }^{341}$

339 BMBF 2002: Strategisches Positionspapier des Bundesministeriums für Bildung und Forschung zur Zukunft der wissenschaftlichen Informationen Deutschland. Information vernetzen- Wissen aktivieren. https://bit.ly/30kh8Vc.

Nicht minder interessant ist hier das zweite strategische Ziel im BMBF-Positionspapier: „Den durch die Entwicklung moderner Informations- und Kommunikationstechnologien und Netze ermöglichten Paradigmenwechsel vom Anbieter zum Nutzer im Hinblick auf individuelle Informationsanforderungen vollziehen”. Vgl. Bundesregierung Masterplan 03.12.2003 - https://bit.ly/2m2ueXP.

340 Bundesregierung 2007 Zweiter Korb - https://bit.ly/2ZhNnTA.

341 Eine Verfassungsbeschwerde gegen die Streichung von $\S 31$ Abs. 4 UrhG wurde vom BVerfG nicht zur Entscheidung - in erster Linie aus formalen Begründen angenommen - Beschluss vom 
Die Bundesregierung hatte in der Begründung für ihren RegE $E^{342}$ allerdings durchaus die Gefahr gesehen, dass „der Urheber ... dem Verwerter als dem stärkeren Vertragspartner ... schutzlos ausgeliefert“ sein könnte. Daher darf diese Rechteübertragung nicht automatisch geschehen. Der neu eingefügte $\S 31 \mathrm{a}$ Abs. 1 sieht vor, dass ein Vertrag über die Einräumung von Nutzungsrechten für unbekannte Nutzungsarten der Schriftform bedürfe. ${ }^{343}$ Das hat wohl die praktische Konsequenz, dass seitdem Verlage diese Erweiterung in ihren Vertragstexten einfach aufnehmen, und so gut wie alle Autoren dies dann so gegenzeichnen, ohne dass sie sich der Konsequenzen bewusst sind. Zudem erhält der Urheber „neben dem obligatorischen Vergütungsanspruch für neue Nutzungsarten auch ein Widerrufsrecht“. Für diesen Widerspruch hat der Urheber drei Monate Zeit, dem zu widersprechen. Allerdings kann kaum gesichert werden, dass den Urheber diese Mitteilung erreicht. Sie muss nur an die dem Verlag „zuletzt bekannte Adresse abgesendet“ werden. „Demnach unerheblich ist, ob die Mitteilung dem Urheber auch tatsächlich zugeht. Dieser trägt ... vielmehr das alleinige Übertragungsrisiko." ${ }^{344}$ Für den Verlag reicht die dokumentierte Absendung der Mitteilung. Kein Einschreiben nötig. Das Widerrufsrecht gilt auch nicht, „wenn sich die Parteien nach Bekanntwerden der neuen Nutzungsart auf eine Vergütung nach §32c Abs. 1 geeinigt haben“ (§31a Abs. 2). Fraglich ob durch dieses „Widerrufsrecht“ die Asymmetrie zwischen den Autoren und den wirtschaftlich stärkeren Verwertern” vermindert werden kann. ${ }^{345}$

Verlage sind daran interessiert, ihr Angebotsportfolio dadurch zu erweitern, dass schon analog publizierte Werke nun auch in digitalisierter Form angeboten werden oder dass aus Teilen verschiedener Werke neue Produkte erstellt und/oder über bislang nicht bekannte Vertriebswege auf den Markt gebracht werden. Sie sind auch daran interessiert, für diese neuen Verwertungen exklusive

\footnotetext{
24. November 2009 - 1 BvR 213/08. Zum Beschluss des BVerfG (Wille 2009) Anmerkung zu BVerfG, Beschluss vom 24. November 2009. Wille macht allerdings deutlich, dass das BVerfG das Problem Streichung von $\S 31$ Abs. 4 für ,grundrechtlich nicht gelöst hält” (243).

342 RegE Zweiter Korb, Drucksache16/1828,15.6.2006 - https://bit.ly/2lIatoa.

343 Allerdings wird das durch $\S 31$ a Abs. 2 Satz 2 eingeschränkt: „Der Schriftform bedarf es nicht, wenn der Urheber unentgeltlich ein einfaches Nutzungsrecht für jedermann einräumt.“

344 (Schwarz 2018) Die Beendigung urheberrechtlicher Nutzungsrechte, 221.

345 „Nach §31a Abs. 1 S. 2 UrhG-E besteht das Widerrufsrecht des Urhebers nur, solange der andere noch nicht begonnen hat, das Werk in der neuen Nutzungsart zu nutzen. Hierbei besteht keine Verpflichtung des Verwerters, den Urheber über die Entstehung einer neuen Nutzungsart zu informieren. Es ist daher in einer Vielzahl von Fällen zu erwarten, dass der Urheber von einer neuen Nutzungsart nichts erfährt oder zumindest erst dann etwas davon erfährt, wenn die wirtschaftlich erfahrenere Seite längst mit der Nutzung begonnen und der Urheber sein Widerrufsrecht dadurch bereits verloren hat. Das Leerlaufen des Widerrufsrechts ist bei dieser Ausgestaltung bereits vorprogrammiert.“- (Hoeren/Köcher 2005) S. $13 \mathrm{f}$.
} 
Nutzungs-/Verwertungsrechte gegenüber den Urhebern und neue Vergütungsansprüche gegenüber Nutzern von in irgendeiner Form neu aufbereiteten Werke durchsetzen zu können. Entsprechend wollte der Gesetzgeber Verlage von der aufwändigen Aufgabe entpflichten, für neue, zur Zeit des ursprünglichen Vertrags noch unbekannte bzw. nicht explizit im Vertrag erwähnte Verwertungsformen bei allen beteiligten Urhebern oder deren Rechtsnachfolgern um Erlaubnis nachzusuchen. ${ }^{346}$

Es ist durchaus im Sinne auch der Öffentlichkeit, dass erweiterte Dienstleistungen und neue Nutzungsformen für früher schon publizierte Werke bereitgestellt werden. Tatsächlich sind mit den unbekannten Nutzungsarten (unbekannt zum Zeitpunkt der Erstverwertung eines Werks) die Möglichkeiten gemeint, die sich durch die Entwicklung der Technik allgemein und gegenwärtig durch die Digitalisierung von analogen Archivbeständen ergeben haben. Neue Nutzungsarten werden nicht alleine durch technische Rahmenbedingungen festgelegt. ${ }^{347}$ Es muss damit auch eine deutlich andere Verwertungsform, also mit einem anderen Geschäftsmodell als es vorher angewendet wurde, verbunden sein. Die Wiedergabe eines bis dahin gedruckten Buches nun über ein E-Book ist keine neue Nutzungsart - möglicherweise aber der Vertrieb dieses E-Books über eine selbständige Lizenzierungsplattform. Entscheidend für die Anerkennung als neue Nutzungsart sollte nicht nur das Kriterium der ökonomischen Verwertbarkeit dieser durch Technik ermöglichten Verwertung sein, sondern auch, ob diese neue Nutzungsart für die Endnutzer tatsächlich zu einem neuen Nutzen führt und wenn dies mit dem allgemeinen Interesse der Öffentlichkeit an einer erweiterten Nutzungsart zusammengeht.

Ob diese bis heute geltende Reform durch Streichung eines Autorenrechtes dem öffentlichen Interesse an neuen Nutzungsformen gerecht wird, kann bezweifelt werden. Hier wird die Position vertreten, dass die Zweckübertragungsregel ${ }^{348}$ strikt zur Anwendung kommen und eine bislang unbekannte Nutzungsart auf ein einfaches Nutzungsrecht beschränkt werden sollte (so auch Spindler 2008, 10). Dabei könnte vorgesehen werden, dass die Verlage, die das erste einfache Nutzungsrecht nach der Zweckübertragungsregel erworben haben, Vorrang gegenüber anderen Verwertern für die Verhandlungen mit dem Urheber haben sollen. Im Übrigen ist

346 Vgl. (Dreier/Schulze 2018), S. 503, RN 1.

347 (Dreier/Schulze 2018) weisen zu Recht darauf hin, dass die Bestimmung, was eine neue Nutzungsart ist, nicht nur von der Technik abhängt: „Lediglich technische Neuerungen, die eine neue Verwendungsform kennzeichnen, ohne wirtschaftlich eigenständige Vermarktungsmöglichkeiten zu erschließen, sollen nicht ausreichen, um eine neue Nutzungsart annehmen zu können.“ (UrhG §31a Rn. 35-40 Technischer Fortschritt, Substitution).

348 Entsprechend $\S 31$ Abs. 5 UrhG: „Sind bei der Einräumung eines Nutzungsrechts die Nutzungsarten nicht ausdrücklich einzeln bezeichnet, so bestimmt sich nach dem von beiden Partnern zugrunde gelegten Vertragszweck, auf welche Nutzungsarten es sich erstreckt.“ 
bis heute nicht zu erkennen, ob diese Reform wirklich zu Innovationen der Verlage für neue Produkte mit informationellen Mehrwerteigenschaften geführt hat. Für die meisten alten Publikationen wird es sich um vergriffene Werke handelt. Für deren Wiederauflage im elektronischen Format hat der Gesetzgeber 2013 mit der Regelung für vergriffene Werke gesorgt (vgl. 11.2).

\subsection{Fazit zu den Schrankenregelungen des Ersten und Zweiten Korbs}

Nicht nur die Regulierungen im enttäuschenden Ersten Korb (vor allem durch §52a) sondern auch die durch den Zweiten Korb auf die Bibliotheken bezogenen Schrankenregelungen der $\S \S 52 \mathrm{~b}$ und 53a haben gezeigt, dass die Nutzungsmöglichkeiten für die Akteure in Bildung und Wissenschaft so stark eingeschränkt wurden, dass sie eher der Wahrung der Verlagsinteressen dienten, nicht aber denen von Bildung und Wissenschaft. Dies hat quasi zu einer kuriosen Neubestimmung des Schrankenbegriffs geführt. Schranken sollten die exklusiven Rechte der Rechtsinhaber einschränken. Die Schrankenbestimmungen in den beiden Körben der Urheberrechtsreform führten eher zu Einschränkungen der Nutzungsrechte, wie sie jahrzehntelang im analogen Umfeld üblich waren, obgleich die Digitalisierung eher eine freiere Nutzung begünstigen könnte/sollte. Die Einschätzung, dass die mit Blick auf Bildung und Wissenschaft abzielenden Schrankenregelungen in der Gänze und in vielen Details sich als wenig unterstützend für Bildung und Wissenschaft erwiesen haben, hat in der Fachwelt breite Zustimmung gefunden. ${ }^{349}$

349 Vgl. die Einschätzung der Allianz der Wissenschaftsorganisationen in ihrem Kommentar zu der Entfristung von § 52a vom 1.12.2014: „,nach Auffassung der Allianz nicht darüber hinwegtäuschen, dass sich die bestehenden Schrankenregelungen zugunsten von Bildung und Wissenschaft überwiegend nicht bewährt haben. Die begrifflichen Unsicherheiten und uneinheitlichen Formulierungen entsprechen nicht den Bedürfnissen von Bildung und Wissenschaft und machen es Studierenden, Wissenschaftlerinnen und Wissenschaftlern schwer, die Vorgaben anzuwenden“ https://bit.ly/2MyX7Hp. Am Ende der Dissertation von Bajon über das Wissenschaftsurheberrecht von 2010 die vorsichtige Einschätzung: "Auf einem höheren Abstraktionslevel erscheint die abschließende Einschätzung zulässig, die im Zweiten Korb erfolgten Veränderungen der Wissenschaftsschranken nicht pauschal als Beleg für ein „wissenschaftsfreundliches Urheberrecht“ werten zu können.“ (Bajon 2010) Interessenausgleich im Urheberrecht, 553. de la Durantaye: „Die für Bildung und Wissenschaft relevanten urheberrechtlichen Schranken erfassen in der Regel nur eng umrissene Sachverhalte, sind wenig technologieoffen und nicht allgemeinverständlich formuliert. Zudem sind sie über mehrere Einzelnormen hinweg verstreut. Das führt zu großer Rechtsunsicherheit für Forscher, Wissenschaftler und Lehrer, aber auch für Infrastruktureinrichtungen wie Bibliotheken, Archive und Museen.“ (de la Durantaye 2016) Die Bildungs- und Wissenschaftsschranke, 1. Hoeren/Neubauer: „Es zeigt sich, dass die Regelungen der §§ 52a, 52b 
Auch bei den Diskussionen im Bundestag bei der Verabschiedung des Zweiten Korbs wurde deutlich, dass große Unsicherheit darüber bestand, ob das Ziel eines wissenschaftsfreundlichen Urheberrechts mit den beiden Reformen erreicht wurde. Das galt nicht nur durch für die Kritik der Oppositionsparteien, sondern auch durch die der Regierung selbst. Die Unsicherheit der Politik zeigte sich z. B. auch dadurch, dass der für Bildung und Wissenschaft zentrale §52a befristet wurde und die Befristungen immer wieder verlängert wurden, bis er dann am 6.11.2014, auch auf Grund der Klarstellung einiger Bestimmungen in §52a durch die beiden Urteile des BGH von März und November 2013, viel zu spät und zum damaligen Zeitpunkt auch schon überflüssig ganz entsperrt wurde ${ }^{350}$ - überflüssig, weil die öffentliche und politische Diskussion längst in Richtung einer umfassenden allgemeinen Bildungs- und Wissenschaftsschranke gegangen war. ${ }^{351}$

Thomas Hoeren hat diese Diskussion um die unzureichenden Regelungen des Ersten und Zweiten Korbs bei einem Fachgespräch vor dem Ausschuss Digitale Agenda des Deutschen Bundestages zum Thema „Stand der Urheberrechtsreform auf deutscher und europäischer Ebene” so zusammengefasst:

Die Schranken des §§ 52a, 52b und 53a sind so eng gefasst, dass sie sinnvoll nicht für Unterricht und Wissenschaft genutzt werden können. Eine sinnvolle Nutzung von Werken im Interesse der Wissenschaftsfreiheit ist insofern kaum möglich. Insofern bedarf es einer Generalschranke zu Gunsten von Wissenschaft und Forschung (hier ähnlich wie im Bereich Fair Use).

und 53a UrhG in vielfacher Hinsicht überarbeitungsbedürftig sind.“ (Hoeren/Neubauer 2012) Zur Nutzung urheberrechtlich geschützter Werke in Hochschulen und Bibliotheken, 642. Aus der Blog-Welt: „Als missglückt wird von Wissenschafts- und Bibliotheksvereinigungen sowie vom Bundesrat die von der großen Koalition angestrebte Schaffung eines wissenschaftsfreundlichen Urheberrechts bezeichnet.“- (Krempl 2007) Neues Urheberrecht tritt Anfang 2008 in Kraft https://bit.ly/2TvUOrh.

350 Die Aufhebung der Befristung von §52a ist im Zusammenhang der beiden Urteile des BGH von März und November 2013 zu sehen. Durch diese Urteile wurden einige der offenen Fragen, die nicht zuletzt durch die Verwendung unbestimmter Begriffe („kleine Teile von Werken“ etc.) entstanden waren, verbindlich beantwortet. §52a wurde dadurch nicht grundlegend besser, aber zumindest für dessen Anwendung praxisfester und rechtssicherer (vgl. $10.1 \mathrm{zu} \S 52 \mathrm{a})$.

351 Der Deutsche Bundestag hat entsprechend einem Antrag der CDU/CSU- und SPD-Fraktionen auf Entfristung von § 52a schließlich am 25.9.2014 in ersten Lesung zugestimmt, allerdings mit dem Hinweis, dass die politische Zielsetzung der umfassenden Bildungs- und Wissenschaftsschranke nicht durch 52a als erfüllt angesehen werden kann, und schon gar nicht präjudiziere die Regelung des $\S 52 a$,die Einführung einer einheitlichen Bildungs- und Wissenschaftsschranke. [...] Die umfassende Umgestaltung aller Schrankenregelungen in diesem Bereich erfordert eine intensive rechtspolitische Diskussion, die voraussichtlich nicht vor Ende der Befristung des §52a [Ende 2014 - RK] abgeschlossen werden kann.“ - Gesetzentwurf der Fraktionen der CDU/CSU und SPD Entwurf eines Gesetzes zur Änderung des Urheberrechtsgesetzes. Drucksache 18/2602, 23.09.2014 https://bit.ly/2m4uRjJ. 
